\title{
Efficacy of Blood Sources and Artificial Blood Feeding Methods in Rearing of Aedes aegypti (Diptera: Culicidae) for Sterile Insect Technique and Incompatible Insect Technique Approaches in Sri Lanka
}

\author{
Nayana Gunathilaka,' Tharaka Ranathunge, ${ }^{2}$ \\ Lahiru Udayanga, ${ }^{2}$ and Wimaladharma Abeyewickreme ${ }^{3}$ \\ ${ }^{1}$ Department of Parasitology, Faculty of Medicine, University of Kelaniya, Ragama, Sri Lanka \\ ${ }^{2}$ Molecular Medicine Unit, Faculty of Medicine, University of Kelaniya, Ragama, Sri Lanka \\ ${ }^{3}$ Dengue Mega Project, Faculty of Medicine, University of Kelaniya, Ragama, Sri Lanka \\ Correspondence should be addressed to Nayana Gunathilaka; n.gunathilaka@kln.ac.lk
}

Received 21 January 2017; Revised 26 May 2017; Accepted 17 July 2017; Published 15 August 2017

Academic Editor: Cheng-Feng Qin

Copyright (C) 2017 Nayana Gunathilaka et al. This is an open access article distributed under the Creative Commons Attribution License, which permits unrestricted use, distribution, and reproduction in any medium, provided the original work is properly cited.

\begin{abstract}
Introduction. Selection of the artificial membrane feeding technique and blood meal source has been recognized as key considerations in mass rearing of vectors. Methodology. Artificial membrane feeding techniques, namely, glass plate, metal plate, and Hemotek membrane feeding method, and three blood sources (human, cattle, and chicken) were evaluated based on feeding rates, fecundity, and hatching rates of Aedes aegypti. Significance in the variations among blood feeding was investigated by one-way ANOVA, cluster analysis of variance (ANOSIM), and principal coordinates (PCO) analysis. Results. Feeding rates of Ae. aegypti significantly differed among the membrane feeding techniques as suggested by one-way ANOVA $(p<0.05)$. The metal plate method was identified as the most efficient and cost-effective feeding technique. Blood feeding rate of Ae. aegypti was higher with human blood followed by cattle and chicken blood, respectively. However, no significant difference was observed from the mosquitoes fed with cattle and human blood, in terms of fecundity, oviposition rate, and fertility as suggested by one-way ANOVA $(p>0.05)$. Conclusions. Metal plate method could be recommended as the most effective membrane feeding technique for mass rearing of $A e$. aegypti, due to its high feeding rate and cost effectiveness. Cattle blood could be recommended for mass rearing Ae. aegypti.
\end{abstract}

\section{Background}

Dengue is the most rapidly spreading mosquito-borne viral disease in the world, which is mainly transmitted by Aedes aegypti mosquitoes [1]. During 2016, 54,364 suspected dengue cases have been reported to the Epidemiology Unit, Sri Lanka, from all over the island indicating the highest ever number of cases per year. Approximately, over $49 \%$ of dengue cases have been reported in 2016 from the Western province, Sri Lanka [2]. Absence of effective drugs and vaccines for dengue virus remains as the major issue in elimination of dengue from the country. Therefore, the main focus of the disease controlling entities is to control the vector densities of dengue, thereby restricting the transmission of the virus [3].
In Sri Lanka, current vector controlling approaches focus on environmental management, chemical control, and biological control. In view of the problems associated with conventional mosquito control, such as resistance and health effects, new vector control approaches such as sterile insect technique (SIT), incompatible insect technique (IIT), and use of transgenic mosquitoes have been evolved recently $[4,5]$. However, these methods require mass rearing of mosquitoes for laboratory experiments and field based trials.

Nutrition is one of the main factors to be considered in mass rearing of mosquito vectors. Anautogenous female mosquitoes require protein from blood to develop eggs [6]. Laboratory studies on mosquito biology and vector-parasite 
interactions largely require artificial blood delivery systems to feed mosquitoes. Therefore, studies related to effective blood meal sources and artificial blood feeding techniques are essential in establishing laboratory colonies of mosquito vectors [7].

Most of the earlier studies on mosquito rearing relied on human hosts or live animal hosts [8-10]. Currently, live animals such as guinea pigs and rodents are more commonly used. Several different artificial feeders have been developed for feeding of mosquitoes; some are simple; some are more complex.

Various types of membranes are being used in artificial membrane techniques, such as animal tissue, Parafilm-M ${ }^{\circledR}$ films, and collagen membranes [11]. The cattle collagen sausage-casing membrane and Parafilm-M are inexpensively mass customized with quality control. The temperature of the blood meal is generally maintained at $37-39^{\circ} \mathrm{C}$ to attract the mosquitoes to settle [12]. Hemotek membrane feeders (Discovery Workshops, Accrington, UK) use an electric heating element to maintain the temperature of the blood meal [13, 14]. The glass feeder has an outer area with circulating warm water and an inner chamber into which the blood is poured $[15,16]$. However, some of these methods are expensive, time consuming, subjective to regulation, and inspection, thereby limiting their usage in many laboratory settings [7]. On the other hand, some of these facilities are not affordable for some laboratories due to limitations of funds to acquire highly sophisticated apparatus and to bear maintenance cost, especially in the developing countries. Therefore, it is important to develop an inexpensive, convenient, and effective artificial membrane blood feeding method that may also take animal welfare into consideration.

Hence, the objective of the present study was to evaluate the effectiveness of different artificial blood feeding methods and appropriate blood meal source/s for rearing of Ae. aegypti vectors under laboratory conditions in order to facilitate the experiments, which have been implemented on several novel vector control strategies in Sri Lanka. The findings of the current study may encourage laboratories in different institutes which conduct colony maintenance, vector-parasite interactions, and medical entomology related research by seeking inexpensive and effective artificial membrane feeding methods, along with available blood sources that yield the optimum growth and reproductive rates of medically important mosquitoes of their interests.

\section{Methods}

2.1. Establishment of Ae. aegypti Colony. A mosquito colony of Aedes aegypti was established from five blood-engorged wild females caught from the field (Ragama Medical Officer of Health area) and was maintained under a 12:12 (light: dark) cycle. Mosquitoes were reared using standard conditions to generate similar sized individuals. Adult mosquitoes were housed in $24 \times 24 \times 24 \mathrm{~cm}$ mosquito cages with mesh screening on top, provided with a $20 \%$ sugar solution and water ad libitum. The colony was maintained at $27 \pm 2^{\circ} \mathrm{C}$ and $75 \pm 5 \%$ humidity, providing human blood as

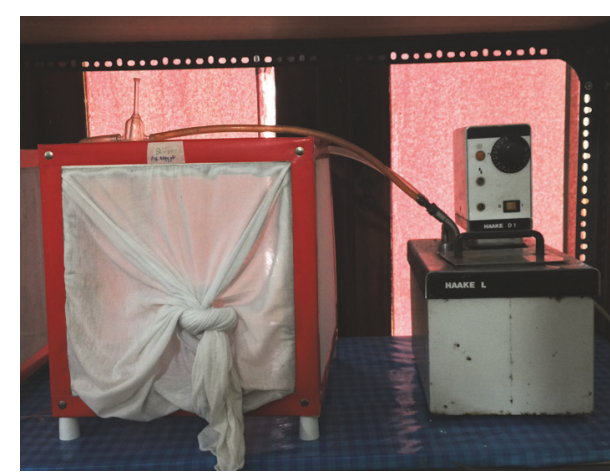

FIgURE 1: Setup of the glass plate method.

the feeding source. A hand-held aspirator was used to access mosquitoes through a tighten opening in one side of the cage. The colony was maintained under the above laboratory settings at the Molecular Medicine Unit, Faculty of Medicine, University of Kelaniya. The offspring from 12th generation of the above colony was used for the current study.

Five-day old females were randomly placed in screen topped oviposition plastic cups $(15 \mathrm{~cm}$ in diameter and $13.5 \mathrm{~cm}$ in depth) with inner side lined with filter paper and filled with approximately $150 \mathrm{ml}$ of filtered tap water. Females were allowed to feed on cattle blood and the eggs laid by them were allowed to be hatched within 2-3 days after oviposition. The first instar larvae $\left(L_{1}\right)$ were transferred daily from the oviposition cups to plastic trays $(40 \times 25 \times 6 \mathrm{~cm})$, containing $2,000 \mathrm{ml}$ of water and reared to the 4 th instar $\left(L_{4}\right)$ larvae with a larval diet containing 50\% tuna meal, 36\% bovine liver powder, and $14 \%$ yeast [17]. The larval rearing trays were examined daily for pupae, which were removed and transferred to mosquito cages for adult emergence.

2.2. Evaluation of the Membrane Feeding Techniques. A batch containing 300 mosquitoes (with 1:1 male: female ratio) of Ae. aegypti in the same age (2-3 days old) was introduced in to $24 \times 24 \times 24 \mathrm{~cm}$ cages using a hand-held aspirator and fed with cattle blood using three different artificial membrane feeding techniques as described below. Blood samples were shaken for 30 seconds at every 30 minutes and the temperature was maintained at $37^{\circ} \mathrm{C}$ to minimize the sedimentation of blood throughout the experiment. After 2 hours of blood feeding, the number of female mosquitoes that successfully fed was recorded and mosquitoes that did not feed or only partially fed were discarded. The procedure was repeated 5 times for each blood feeding technique using the adult Ae. aegypti mosquitoes from the same cohort with similar age as described below.

2.3. Glass Plate Method. A circulating water bath was set to $37^{\circ} \mathrm{C}$ and was connected to holding containers via tubing. A Parafilm-M membrane was stretched across the bottom of the glass tube of the feeder (HAAKE, Model 000-3350), with a surface area of $7.07 \mathrm{~cm}^{2}$, and secured with a rubber band. Blood was added $(3.0 \mathrm{ml})$ to the funnel of the glass feeder (Figure 1). 

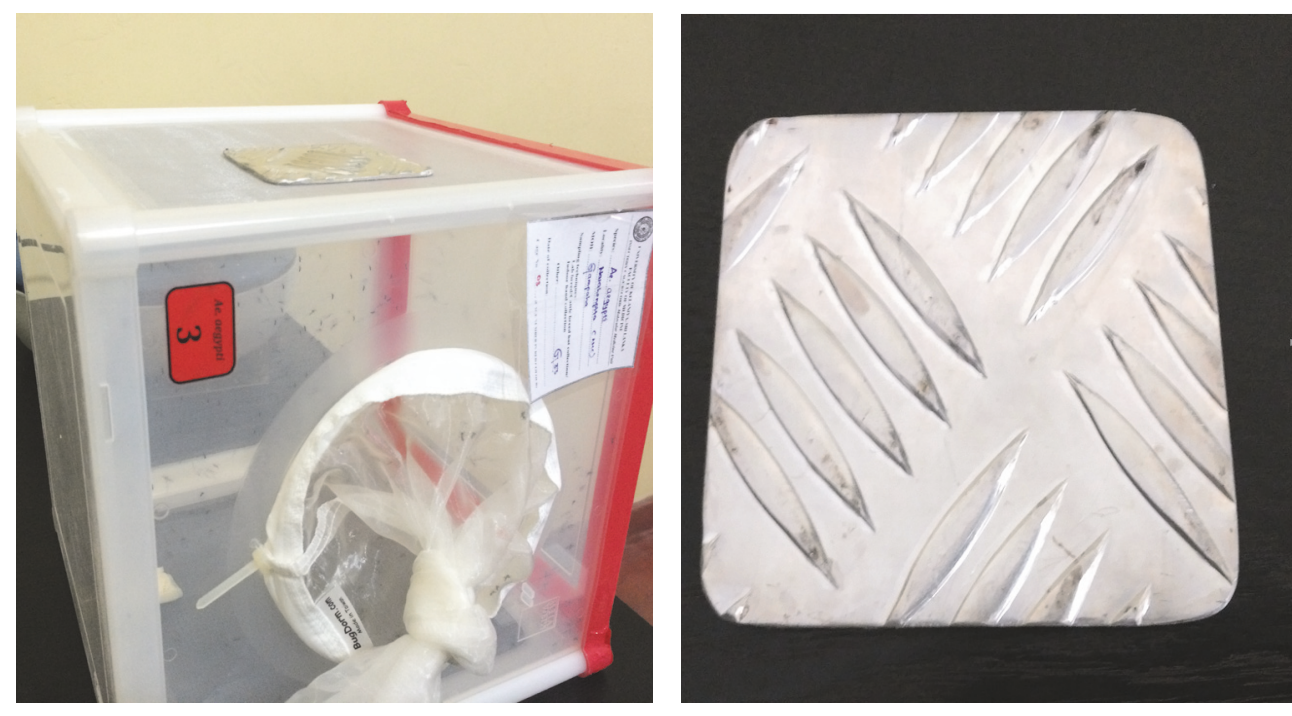

FIGURE 2: Metal plate and the setup of the metal plate method.

2.4. Metal Plate Method. A square shaped metal plate with a rough surface $(9 \mathrm{~cm} \times 9 \mathrm{~cm}$ : width $\times$ length $)$ was prepared. The rough surface of the developed metal plate was covered with a stretched Parafilm-M membrane leaving one side open. Blood was added $(3.0 \mathrm{ml})$ into the space between the plate and the membrane, and the open side was carefully sealed. A small pillow $(10 \mathrm{~cm} \times 10 \mathrm{~cm}$ : width $\times$ length $)$ filled with rice grains was heated up to $40^{\circ} \mathrm{C}$ using a microwave oven and was placed on top of the metal plate, while keeping blood filled surface down in order to maintain the temperature throughout the time of blood feeding (Figure 2).

\subsection{Hemotek Membrane Feeding Method. A Parafilm-M} membrane was stretched across the bottom of the Hemotek membrane blood feeder (PS-6 System, Discovery Workshops, Accrington, UK) having a surface area of $9.62 \mathrm{~cm}^{2}$. Blood $(3.0 \mathrm{ml})$ was transferred into the Hemotek blood reservoir unit and the temperature was set to $37^{\circ} \mathrm{C}$ (Figure 3).

2.6. Evaluation of Blood Meal Success by Ae. aegypti. Three different blood meal sources, namely, human, cattle, and chicken, were taken for the study. Both cattle and chicken blood were purchased from a slaughter house (acquired from a healthy individual), which is operated in accordance with standard protocols under the supervision of Public Health Inspectors (PHI). Human blood was obtained from a healthy volunteer ( $<18$ years) after obtaining the written consent from the donor. The blood was taken under sterile condition by a trained technical officer under supervision of a Medical Officer at the Faculty of Medicine, University of Kelaniya, Sri Lanka.

The blood samples were stored at $4^{\circ} \mathrm{C}$ after adding ethylene diamine tetraacetic acid (EDTA) as an anticoagulant (EDTA : blood $=1: 100)$ until taken for experiments. Prior to the experimental trials, blood samples were kept at room temperature for 30 minutes and introduced to the female

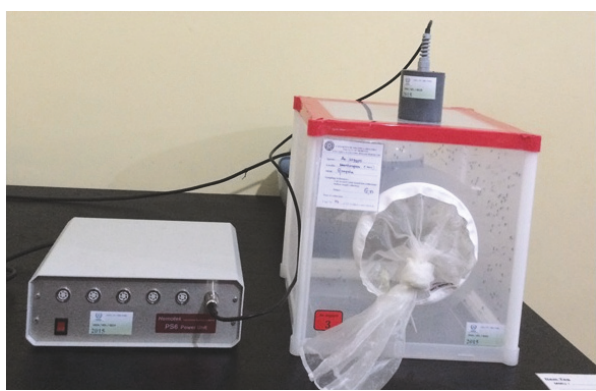

FIgURE 3: Setup of the Hemotek membrane feeding system.

mosquitoes in holding containers using metal plate method as described above.

Batches of 300 Ae. aegypti mosquitoes (with 1:1 male to female ratio) were fed with blood using metal plate method, as described above. After feeding of blood, the mosquitoes were transferred into screen topped oviposition plastic cups $(15 \mathrm{~cm}$ in diameter and $13.5 \mathrm{~cm}$ in depth) with inner side lined with filter paper and filled with approximately $150 \mathrm{ml}$ of filtered tap water. Ae. aegypti mosquitoes were allowed to mate naturally till two days after blood feeding within the container. After 2 days, the filter paper lining within each container was taken out and were allowed to dry for 7 days. The number of eggs on each paper was enumerated under a light microscope by trained personals along with accepted quality control procedures.

Subsequently, eggs were allowed to be hatched separately and the first instar larvae $\left(L_{1}\right)$ that emerged from each filter paper lining were enumerated, separately for 2 days after introduction of the egg papers into the hatching bottles. The whole procedure was repeated for 5 times for each blood source.

2.7. Data Interpretation and Statistical Analysis. Blood feeding rates of female mosquitoes were calculated for each 
TABLE 1: Mean number of female mosquitoes that fed from different feeding methods, blood feeding rate along with the results of General Linear Modelling (number of replicates $[n]=5$ ).

\begin{tabular}{|c|c|c|c|}
\hline Feeding technique & Number of females & Number of blood fed & Blood feeding rate (\%) \\
\hline Metal plate & 300 & $\begin{array}{c}296 \pm 2.33 \\
(293.67-298.33)\end{array}$ & $\begin{array}{l}98.67 \pm 0.77^{\mathrm{a}} \\
(97.90-99.44)\end{array}$ \\
\hline Hemotek membrane feeding method & 300 & $\begin{array}{c}271 \pm 2.33 \\
(268.67-273.33)\end{array}$ & $\begin{array}{l}90.33 \pm 0.77^{\mathrm{b}} \\
(97.90-99.44)\end{array}$ \\
\hline Glass plate & 300 & $\begin{array}{c}251 \pm 5.29 \\
(245.71-256.29)\end{array}$ & $\begin{array}{l}83.67 \pm 1.76^{\mathrm{c}} \\
(81.91-85.43)\end{array}$ \\
\hline
\end{tabular}

Note. Values are mean \pm SE, range in parenthesis. Different superscript letters in a column show significant differences $(p<0.05)$ as suggested by General Linear Modelling followed by Tukey's HSD (Honest Significant Difference) at 95\% level of significance.

TABlE 2: Mean percentage of female mosquitoes that fed with different blood types, blood feeding rates, their egg laying efficiency, egg hatching rate, and the results of General Linear Modelling (number of replicates $[n]=5$ ).

\begin{tabular}{|c|c|c|c|c|c|c|c|}
\hline $\begin{array}{l}\text { Type of } \\
\text { blood }\end{array}$ & $\begin{array}{l}\text { Number of } \\
\text { females }\end{array}$ & $\begin{array}{l}\text { Number of } \\
\text { blood fed } \\
\text { females }\end{array}$ & $\begin{array}{l}\text { Blood feeding } \\
\text { rate }(\%)\end{array}$ & Fecundity & $\begin{array}{l}\text { Egg laying effi- } \\
\text { ciency/oviposition } \\
\text { rate }\end{array}$ & $\begin{array}{c}\text { Number of eggs } \\
\text { hatched }\end{array}$ & $\begin{array}{l}\text { Egg hatching } \\
\text { rate }(\%)\end{array}$ \\
\hline Cattle & 300 & $\begin{array}{c}270 \pm 2.89^{\mathrm{a}} \\
(267.11-272.89)\end{array}$ & $\begin{array}{l}89.67 \pm 0.96^{a} \\
(88.71-90.63)\end{array}$ & $\begin{array}{c}4196 \pm 39.10^{\mathrm{a}} \\
(4156.90-4235.10)\end{array}$ & $\begin{array}{l}15.54 \pm 0.31^{\mathrm{a}} \\
(15.23-15.85)\end{array}$ & $\begin{array}{c}3904 \pm 26.30^{\mathrm{a}} \\
(3877.70-3930.30)\end{array}$ & $\begin{array}{l}93.04 \pm 0.25^{\mathrm{a}} \\
(92.79-93.29)\end{array}$ \\
\hline Human & 300 & $\begin{array}{c}292 \pm 2.93^{\mathrm{b}} \\
(289.07-294.93)\end{array}$ & $\begin{array}{c}97.33 \pm 0.99^{\mathrm{b}} \\
(96.34-98.32)\end{array}$ & $\begin{array}{c}4336 \pm 45.25^{\mathrm{a}} \\
(4290.75-4381.25)\end{array}$ & $\begin{array}{l}14.85 \pm 0.39^{\mathrm{a}} \\
(14.46-15.24)\end{array}$ & $\begin{array}{c}4064 \pm 40.9^{\mathrm{a}} \\
(4023.10-4104.9)\end{array}$ & $\begin{array}{c}93.73 \pm 0.75^{\mathrm{a}} \\
(92.98-94.48)\end{array}$ \\
\hline Chicken & 300 & $\begin{array}{c}239 \pm 2.73^{\mathrm{c}} \\
(236.27-241.73)\end{array}$ & $\begin{array}{l}79.67 \pm 0.91^{\mathrm{c}} \\
(78.76-80.58)\end{array}$ & $\begin{array}{c}3034 \pm 34.90^{\mathrm{b}} \\
(2999.10-3068.9)\end{array}$ & $\begin{array}{l}12.69 \pm 0.28^{\mathrm{b}} \\
(12.41-12.97)\end{array}$ & $\begin{array}{c}2681 \pm 33.70^{\mathrm{b}} \\
(2647.30-2714.7)\end{array}$ & $\begin{array}{l}88.39 \pm 2.65^{\mathrm{a}} \\
(85.74-91.04)\end{array}$ \\
\hline
\end{tabular}

Note. Values are mean \pm SE, range in parenthesis. Different superscript letters in a column show significant differences $(p<0.05)$ as suggested by General Linear Modelling followed by Tukey's HSD (Honest Significant Difference) test, at 95\% level of significance.

membrane feeding method and blood meal source, separately. Fecundity of the female mosquitoes that were fed with different sources of blood was calculated as the number of eggs laid by the females. The oviposition rate for females was calculated as the percentage of eggs laid by fully engorged females, while fertility was calculated as the percentage of larvae that emerged after hatching as recommended by Clements, 1992 [6].

The SPSS (version 21) and Plymouth Routines in Multivariate Ecological Research version 6 (PRIMER 6) were devised to perform the statistical comparisons. In case of nonnormal distributions, normalization and square root transformation techniques in PRIMER 6 were employed before processing of data. Significance in the variations among blood feeding rates of three membrane feeding techniques and variations among blood feeding rates, fecundity, and egg hatching rate for each blood meal source were evaluated using General Linear Modelling followed by Tukey's HSD (Honest Significant Difference). Further, cluster analysis (with Bray Curtis resemblance), analysis of variance (ANOSIM), and principal coordinates (PCO) were used to investigate the effect of different blood sources on the investigated parameters cumulatively.

\section{Results}

3.1. Evaluation of Membrane Feeding Techniques. Mean number of female mosquitoes that fed from different feeding methods and the blood feeding rates of females for each membrane feeding technique are presented in Table 1, along with the results of General Linear Modelling. The highest blood feeding rate of Ae. aegypti was identified from metal plate method $(98.67 \%, n=296)$ followed by Hemotek method $(90.33 \%, n=271)$ and glass plate method $(83.67 \%$, $n=251)$. Therefore, metal plate method can be identified as the most efficient blood feeding method for Ae. aegypti ( $p<0.05$ at $95 \%$ level of confidence).

3.2. Evaluation of Blood Source Success of Ae. aegypti. Mean percentage of female mosquitoes that fed with different sources of blood, fecundity, their egg laying efficiency, and egg hatching rate are presented in Table 2 along with the results of General Linear Modelling. Among the three blood meal sources tested, human blood was identified as the most preferred blood source (Table 2) by Ae. aegypti followed by cattle and chicken blood in terms of both number of blood fed females and blood feeding rate $(p<0.05$ at 95\% level of significance). However, the fecundity, egg laying efficiency, and eggs hatching rate were not indicating any significant differences among human and cattle origin blood sources according to the results of General Linear Modelling followed by Tukey's pair wise comparison (Table 2 ).

The overall clustering status of different blood sources in terms of blood feeding rate, fecundity, egg laying efficiency, and egg hatching rate of Ae. aegypti is illustrated in Figure 4. As suggested by the Bray Curtis similarity clustering, both human and cattle blood indicated a high similarity of $98.17 \%$, while chicken blood indicated a dissimilarity of $17.81 \%$ with other two sources based on the cumulative effect of blood feeding rate, fecundity, egg laying efficiency, and eggs hatching rate. The global $R$ value of 0.97 gained for the analysis of similarities (ANOSIM) also confirmed the above 


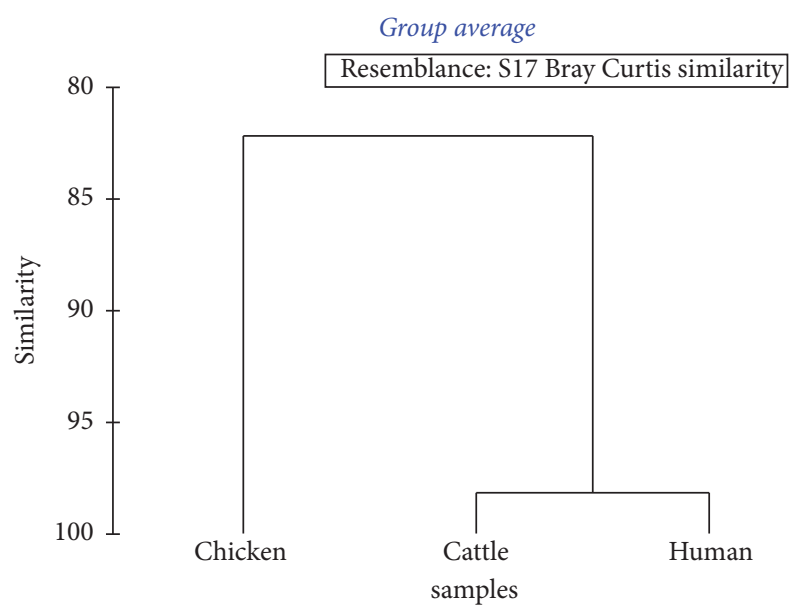

Figure 4: Dendrogram for the cluster analysis of blood source success by Ae. aegypti.

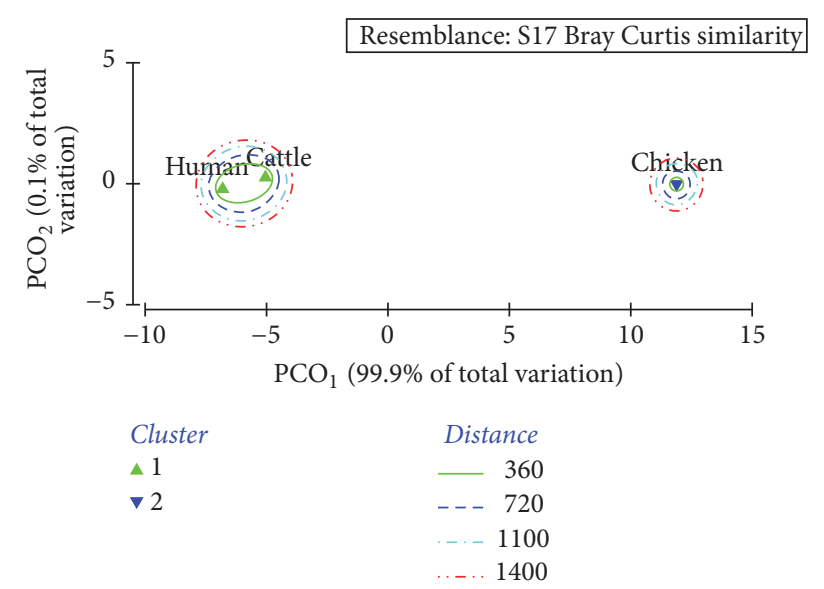

FIgURE 5: Ordination of the different blood sources on $\mathrm{PCO}_{1}$ and $\mathrm{PCO}_{2}$ scores of PCO analysis in terms of success of Ae. aegypti.

observation at a significance level of $5 \%$. Meanwhile, both $\mathrm{PC}_{1}$ and $\mathrm{PC}_{2}$ axes of the principal coordinates (PCO) that accounted for the total variation (100\%) of the blood source success of Ae. aegypti (Figure 5) suggested the emergence of two major clusters as human and cattle blood together, while chicken blood remained isolated. Hence, the results of oneway ANOVA are further confirmed by the cluster and PCO analytical procedures.

\section{Discussion}

Many researches are involved in developing innovative vector control approaches along with conventional vector control methods. However, these approaches need laboratory experiments and artificial rearing of vectors in mass scale. In order to achieve this, it is essential to provide necessary conditions for them to grow, rather in an anthropogenic environment. Many epidemiologically important issues revolve around the feeding behaviour of vector mosquitoes [18]. Selection of a suitable blood meal source and cost-effective technique are the main challenges at insectary setup. Membrane blood feeding system as an artificial feeder to blood-feed Aedes mosquitoes is unique and suitable for multiple purposes. It is important to develop an inexpensive, convenient, and effective artificial membrane blood feeding technique that takes animal welfare into consideration.

Artificial feeding systems that have been developed differ with respect to the composition of the meal, the nature of the membrane, and the method of temperature regulation [14]. Several types of liquid diets including goose, chicken, bovine, pig, mice, rat, gerbil, hamster, rabbit, nonhuman primate blood types, and artificial diets are being used for the purpose of blood feeding mosquitoes [16].

A previous study with blood fed Ae. aegypti indicated the highest fecundity by avian blood than mammalian blood [19]. Conversely, Suleman and Shirin [20] showed that mammalian blood results in greater fecundity than avian blood in Culex quinquefasciatus. These studies demonstrate variations in fecundity between species fed with different blood sources [19-21]. Despite fecundity differences, no variation was seen in fertility (number of larvae hatched from eggs) for $C x$. quinquefasciatus provided with different blood sources [20].

According to the available literature, Ae. aegypti prefers mammalian hosts [22] and may preferentially feed on humans, even in the presence of alternative hosts [23]. It is evidenced the Aedes mosquitoes feed multiple times during one gonotrophic cycle [24], which may facilitate the disease transmission. However, the host preference of Ae. aegypti in Sri Lanka is not well understood, since there is no study conducted to evaluate the hematophagous tendencies among wild population. Therefore, the studies on above aspects should be given high priory in Sri Lanka preceding to the release of transgenic or sterile mosquitoes in controlling dengue vectors.

The present study indicated that the blood meal source affects feeding rates and reproduction in colonized $A e$. aegypti under the laboratory conditions. Mosquitoes fed on human blood demonstrated higher feeding rates, fecundity, oviposition, and fertility rates than those fed with other two (cattle and chicken) blood meal sources. However, obtaining human blood for insectary colony maintenance is problematic due to several ethical and safety issues. In addition, human blood is very scares, since there are limited donors. Human blood stored at the blood banks mainly provides blood for medical emergencies rather than experimental work at insectaries.

Therefore, selection of a blood meal source which is fairly efficient and readily available compared to human blood is essential in rearing. Some studies evidenced that the chicken blood contains nucleated blood cells, while mammalian blood contains anucleated cells, which may influence fecundity if nucleated cells contain more nutrition [20,25]. However, the present study revealed the lowest fecundity from chicken blood.

As there were no significant differences among mosquitoes fed with cattle and human blood in terms of fecundity, oviposition rate, and fertility, the cattle blood may be suggested to be used as an appropriate blood meal source for rearing of Aedes mosquitoes, even though there 
was a difference between the numerical values with human blood. On the other hand, cattle blood could be easily obtained from farms or slaughter houses that are operated adhering to the government regulations with concerning animal welfare at very low costs or sometimes with no cost. In addition, blood related infections that can be transmitted through cattle blood to the workers accidentally at insectaries will be minimal compared to the use of human blood. Therefore, cattle blood could be recommended as the most convenient blood source to be used under local settings with minimal health risks to coworkers.

The results of the present study demonstrate that the blood meal source may influence physiological processes related to fecundity and fertility in Ae. aegypti. However, further studies are needed to address the extent to which these effects alter interactions between mosquitoes and blood meal sources.

Among the artificial membrane feeding methods, the metal plate method was identified as the most efficient feeding method. Since both Hemotek and glass plate techniques require expensive machinery and electricity, use of metal plate method can be suggested as a cost-effective method to achieve artificial blood feeding for hematophagous insects rearing at laboratory settings.

\section{Conclusion}

Metal plate feeding technique could be recommended as the most effective membrane feeding technique for mass rearing of Aedes aegypti compared to glass plate and Hemotek membrane feeding methods, due to its high feeding rate and cost effectiveness. As there were no significant differences among mosquitoes fed with cattle and human blood in terms of fecundity, oviposition rate, and fertility, the cattle blood may be suggestive to use as an appropriate blood meal source for rearing of Aedes mosquitoes out of other blood meal sources tested (human and chicken), even though there was a difference between the numerical values with human blood.

\section{Disclosure}

Dengue Mega Project is funded by the National Research Council. An earlier version of this research work has been presented as an abstract at the International Postgraduate Research Conference (IPRC) 2016, University of Kelaniya, Sri Lanka.

\section{Conflicts of Interest}

The authors declare that they have no conflicts of interest.

\section{Authors' Contributions}

Nayana Gunathilaka contributed to designing the research, supervision of the research work, and writing the manuscript. Tharaka Ranathunge contributed to conducting laboratory experiments. Lahiru Udayanga contributed to assisting laboratory experiments, statistical analysis, and reviewing the manuscript. Wimaladharma Abeyewickreme contributed to reviewing the manuscript. All authors read and approved the final manuscript.

\section{Acknowledgments}

This work was financially supported by the International Atomic Energy Agency (IAEA RAS 5066) and National Research Council Funded Dengue Mega Grant (NRC TO 1404), Sri Lanka.

\section{References}

[1] P. V. J. Nadeeka, P. A. D. H. N. Gunathilaka, and L. D. Amarasinghe, "Geographic, economic and socio-cultural factors which defining the risk of dengue transmission in Kelaniya, Sri Lanka," Journal of Experimental Biology and Agriculture Sciences, vol. 2, no. 2, pp. 158-164, 2014.

[2] Epidemiology Unit, Ministry of Health, Sri Lanka; Dengue Update, 2016, http://www.epid.gov.lk/web/index.php?option= com_casesanddeaths\&section $=$ trends\&Itemid $=448 \&$ lang $=$ en .

[3] World Health Organization (WHO), Dengue: Guidelines for Diagnosis, Treatment, Prevention And Control, World Health Organization (WHO), Geneva, Switzerland, 2009.

[4] M. R. Wise De Valdez, D. Nimmo, J. Betz et al., "Genetic elimination of dengue vector mosquitoes," Proceedings of the National Academy of Sciences of the United States of America, vol. 108, no. 12, pp. 4772-4775, 2011.

[5] R. V. Gibbons and D. W. Vaughn, "Dengue: an escalating problem," British Medical Journal, vol. 324, no. 7353, pp. 1563$1566,2002$.

[6] A. N. Clements, Biology of Mosquitoes: Development, Nutrition, and Reproduction, vol. 1, CABI Publishing, Wallingford, UK, 1992.

[7] M. J. Turell, "Reduced Rift Valley fever virus infection rates in mosquitoes associated with pledget feedings," American Journal of Tropical Medicine and Hygiene, vol. 39, no. 6, pp. 597-602, 1988.

[8] S. F. Kitchen, W. K. Stratman-Thomas, and M. F. Boyd, "On the relative susceptibility of Anopheles Quadrimaculatus to Plasmodium Vivax and Plasmodium Falciparum," The American Journal of Tropical Medicine and Hygiene, vol. s1-15, no. 4, pp. 485-493, 1935.

[9] L. E. Rozeboom, "The rearing of Anopheles Albimanus wiedemann in the laboratory," The American Journal of Tropical Medicine and Hygiene, vol. s1-16, no. 4, pp. 471-478, 1936.

[10] R. L. Crowell, "Insectary rearing of Anopheles quadrimaculatus: a preliminary report," American Journal of Epidemiology, vol. 32, no. 1, pp. 12-20, 1940.

[11] J. Pothikasikorn, R. Boonplueang, C. Suebsaeng, R. Khaengraeng, and T. Chareonviriyaphap, "Feeding response of Aedes aegypti and Anopheles dirus (Diptera: Culicidae) using out-ofdate human blood in a membrane feeding apparatus," Journal of Vector Ecology, vol. 35, no. 1, pp. 149-155, 2010.

[12] L. Deng, S. Y. Koou, A. B. Png, L. C. Ng, and S. G. Lam-Phua, "A novel mosquito feeding system for routine blood-feeding of Aedes aegypti and Aedes albopictus," Tropical Biomedicine, vol. 26, pp. 169-174, 2012.

[13] H. E. Hagen and J. Grunewald, "Routine blood-feeding of Aedes aegypti via a new membrane," Journal of the American Mosquito Control Association, vol. 6, no. 3, pp. 535-536, 1990. 
[14] J. B. Cosgrove, R. J. Wood, D. Petric, and R. H. Abbott, "A convenient mosquito membrane feeding system," Journal of American Mosquito Control Association, vol. 10, pp. 434-436, 1994.

[15] H. Kasap, D. Alptekin, M. Kasao, A. I. Guzel, and U. Luleyap, "Artificial blood feeding of Anopheles sacharovi on a membrane apparatus," Journal of America Mosquito, vol. 19, pp. 367-370, 2003.

[16] Y.-P. Luo, "A novel multiple membrane blood-feeding system for investigating and maintaining Aedes aegypti and Aedes albopictus mosquitoes," Journal of Vector Ecology, vol. 39, no. 2, pp. 271-277, 2014.

[17] A. Puggioli, F. Balestrino, D. Damiens et al., "Efficiency of three diets for larval development in mass rearing aedes albopictus (Diptera: Culicidae)," Journal of Medical Entomology, vol. 50, no. 4, pp. 819-825, 2013.

[18] N. Gunathilaka, T. Denipitiya, M. Hapugoda, W. Abeyewickreme, and R. Wickremasinghe, "Determination of the foraging behaviour and blood meal source of malaria vector mosquitoes in Trincomalee District of Sri Lanka using a multiplex real time polymerase chain reaction assay," Malaria Journal, vol. 15, no. 1, article 242, 2016.

[19] G. F. Bennett, "The influence of the blood meal type on the fecundity of Aedes (Stegomyia) aegypti L. (Diptera: Culicidae)," Canadian Journal of Zoology, vol. 48, no. 3, pp. 539-543, 1970.

[20] M. Suleman and M. Shirin, "A comparison of the reproductive capacity of Culex quinquefasciatus Say fed on cold- and warmblooded vertebrates," Pakistan Journal of Zoology, vol. 13, pp. 221-228, 1981.

[21] S. L. Richards, S. L. Anderson, and S. A. Yost, "Effects of blood meal source on the reproduction of Culex pipiens quinquefasciatus (Diptera: Culicidae)," Journal of Vector Ecology, vol. 37, no. 1, pp. 1-7, 2012.

[22] M. J. Turell, D. J. Dohm, M. R. Sardelis, M. L. O’Guinn, T. G. Andreadis, and J. A. Blow, "An update on the potential of North American mosquitoes (Diptera: Culicidae) to transmit West Nile virus," Journal of Medical Entomology, vol. 42, no. 1, pp. 57-62, 2005.

[23] R. G. M. Saifur, H. Dieng, A. A. Hassan et al., "Changing domesticity of Aedes aegypti in northern peninsular Malaysia: reproductive consequences and potential epidemiological implications," PLoS ONE, vol. 7, no. 2, Article ID e30919, 2012.

[24] T. W. Scott and W. Takken, "Feeding strategies of anthropophilic mosquitoes result in increased risk of pathogen transmission," Trends in Parasitology, vol. 28, no. 3, pp. 114-121, 2012.

[25] A. E. R. Downe and J. A. Archer, "The effects of different bloodmeal sources on digestion and egg production in Culex tarsalis Coq. (Diptera: Culicidae)," Journal of Medical Entomology, vol. 12, no. 4, pp. 431-437, 1975. 

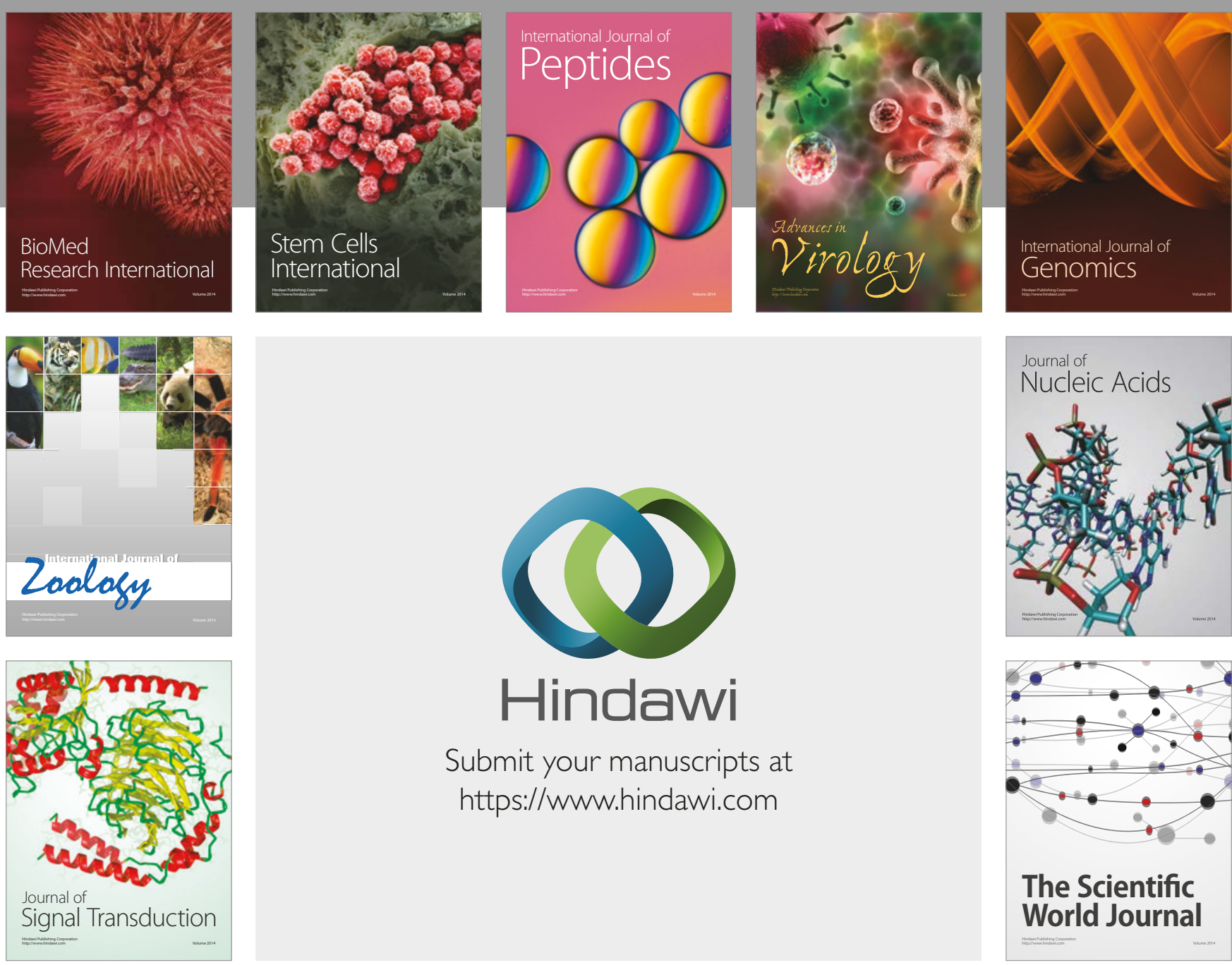

Submit your manuscripts at

https://www.hindawi.com
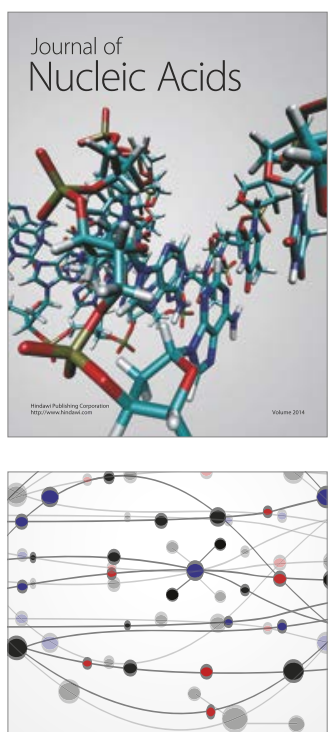

The Scientific World Journal

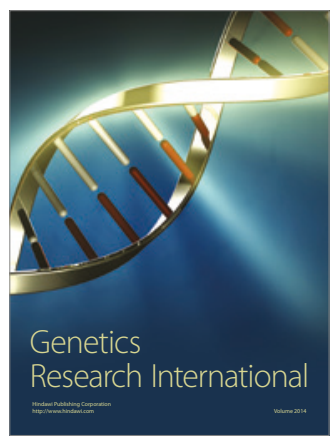

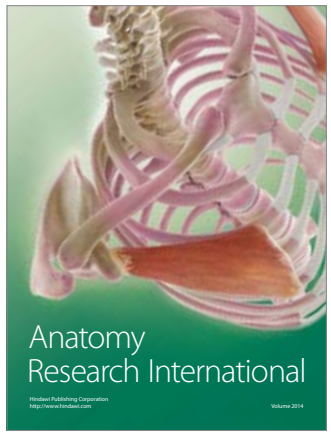

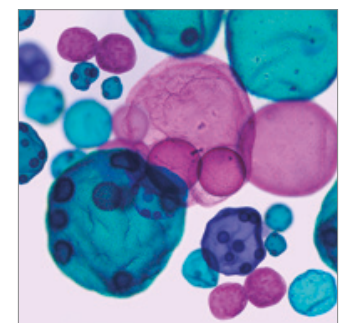

International Journal of Microbiology
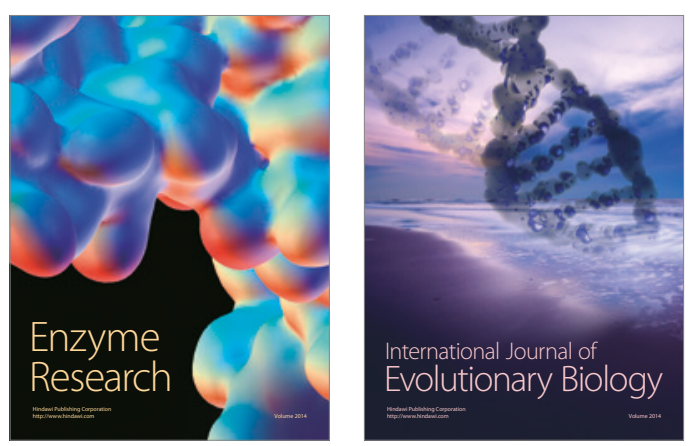
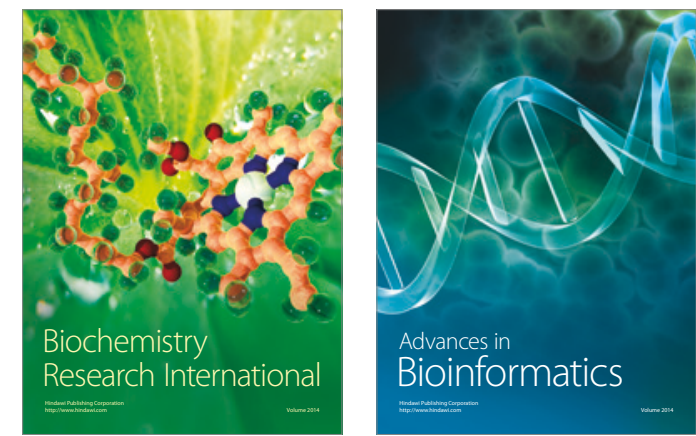

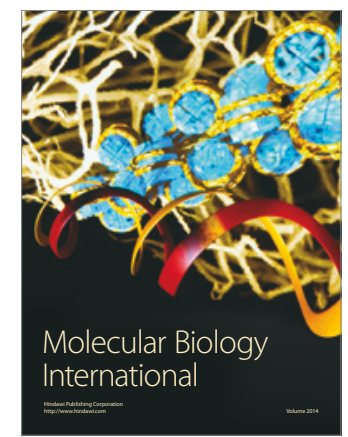

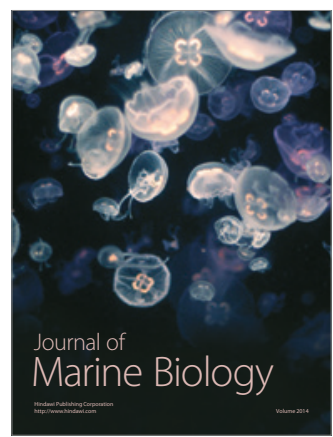

